\title{
Inhibition of P2X7 Purinergic Receptor Ameliorates Cardiac Fibrosis by Suppressing NLRP3/IL-1 $\beta$ Pathway
}

\author{
Junteng Zhou $\left(\mathbb{D},{ }^{1}\right.$ Geer Tian $\left(\mathbb{D},{ }^{2}\right.$ Yue Quan $\mathbb{D}^{2},{ }^{2}$ Junli Li ${ }^{\circ},{ }^{2}$ Xiaojiao Wang $\mathbb{D}^{2}$ \\ Wenchao Wu $\mathbb{D},{ }^{2}$ Miaoling $\mathrm{Li}\left(\mathbb{D},{ }^{3}\right.$ and Xiaojing Liu $\mathbb{D D}^{1,2}$ \\ ${ }^{1}$ Department of Cardiology, West China Hospital, Sichuan University, Chengdu 610041, China \\ ${ }^{2}$ Laboratory of Cardiovascular Diseases, Regenerative Medicine Research Center, West China Hospital, Sichuan University, \\ Chengdu 610041, China \\ ${ }^{3}$ Key Laboratory of Medical Electrophysiology of Ministry of Education, Institute of Cardiovascular Research, \\ Southwest Medical University, Luzhou 646000, China
}

Correspondence should be addressed to Xiaojing Liu; liuxq@scu.edu.cn

Received 20 December 2019; Revised 27 March 2020; Accepted 9 April 2020; Published 22 May 2020

Guest Editor: Ayman M. Mahmoud

Copyright ( 2020 Junteng Zhou et al. This is an open access article distributed under the Creative Commons Attribution License, which permits unrestricted use, distribution, and reproduction in any medium, provided the original work is properly cited.

P2X7 purinergic receptor (P2X7R) has been implicated in several cardiovascular diseases. However, whether it regulates cardiac fibrosis remains elusive. Herein, its involvement in the development of cardiac fibrosis was examined using a transverse aortic constriction (TAC) mice model and cardiac fibroblasts (CFs) hyperstimulated by TGF- $\beta 1$ for 48 hours. Results showed that TAC and TGF- $\beta 1$ treatment increased the expression of P2X7R. Silencing of P2X7R expression with siP2X7R ameliorated TGF$\beta 1$ effects on fibroblasts activation. Similarly, P2X7R inhibition by Brilliant Blue G (BBG) reduced mRNA and protein levels of profibrosis markers, while the P2X7R agonist BzATP accelerated the TGF- $\beta 1$-induced CFs activation. Moreover, it was found that TGF- $\beta 1$-induced CFs activation was mediated by the NLRP3/IL- $1 \beta$ inflammasome pathway. BBG or siP2X7R treatment suppressed NLRP3/IL-1 $\beta$ pathway signaling. In vivo, BBG significantly alleviated TAC-induced cardiac fibrosis, cardiac dysfunction, and NLRP3/IL-1 $\beta$ activation. Collectively, our findings imply that suppressing P2X7R may limit cardiac fibrosis and abnormal activation of CFs.

\section{Introduction}

Myocardial remodeling is a hallmark feature of several cardiovascular diseases $[1,2]$. Cardiac fibrosis is a pathologic condition caused by myocardial remodeling. The condition is characterized by disrupted cardiac morphology, extracellular matrix (ECM) deposition, and impaired cardiac function $[3,4]$. Previous studies have shown that overstimulation of CFs triggers cardiac fibrosis. However, the molecular mechanisms of myocardial fibrosis caused by pressure overload have not been fully elucidated.

Evidence suggests that extracellular nucleotides, such as adenosine triphosphate (ATP), play a role in myocardial fibrosis [5, 6]. Under normal physiological conditions, a small amount of intracellular ATP is in the heart which stimulates erythrocytes, sympathetic nerves, endothelial cells, and skeletal and smooth muscle cells [7]. However, during hypoxia conditions, high concentration of glucose or abnormal shear stress triggers the release of a large amount of ATP into the interstitial space. Excessive extracellular ATP activates other purine signaling pathways leading to the generation of an amplification loop that promotes inflammation causing cellular injuries $[6,8]$.

$\mathrm{P} 2 \mathrm{X}$ receptors are ligand-gated ion channels that respond to extracellular ATP. Seven P2X receptors (P2X1-P2X7) have been identified in the human heart [9]. Among them, P2X7R is the key mediator of inflammation [10]. In Crohn's disease or rheumatoid arthritis, this receptor triggers the production of cytokines associated with inflammation, interleukin-18 or interleukin- $1 \beta$ (IL-1 $\beta$ ), and tumor necrosis factor- $\alpha$ (TNF- $\alpha$ ) [11]. In the failing heart, ATP leakage from injured cells activates P2X7R on the cell membranes leading to the 
stimulation of NOD-like receptor pyrin domain-containing protein 3 (NLRP3). Subsequently, activated NLRP3 facilitates the assembly of a multiprotein complex named "the NLRP3 inflammasome," consisting of NLRP3, an adaptor molecule termed apoptosis-associated speck-like protein containing a CARD domain (ASC) and caspase-1. Activated caspase-1 cleaves a $31 \mathrm{kDa}$ precursor IL- $1 \beta$ (pro-IL-1 $\beta$ ) into $17 \mathrm{kDa}$ mature IL- $1 \beta$. This causes severe inflammatory reactions aggravating cardiac dysfunction and fibrosis $[12,13]$.

Previous works have identified that P2X7R expression promotes myocardial infarction (MI), and inhibition of P2X7R alleviates elevated systolic blood pressure in rats with MI $[14,15]$. Besides, P2X7R inhibitor conferred similar benefits in an experimental model of autoimmune myocarditis (EAM) [16]. However, changes in P2X7R expression and the impact of these changes on cardiac fibrosis induced by pressure overload are not known.

In this study, we postulated that $\mathrm{P} 2 \mathrm{X} 7 \mathrm{R}$ may promote cardiac fibrosis via the NLRP3/IL- $1 \beta$ pathway.

\section{Materials and Methods}

2.1. Animal Procedures, Transverse Aortic Constriction (TAC) Surgery, and Administration of an Antagonist of P2X7R (Brilliant Blue G). All animal experiments were approved by the animal ethics committee of West China Hospital of Sichuan University (ethic number 2014003A). Male C57BL/6 mice (age, 6 weeks) were procured from the Experimental Animal Tech Co. of Weitonglihua (Beijing, China). The TAC procedure is widely performed to create models of cardiac fibrosis due to induced pressure overload [17]. The mice (at 8 weeks of age) were anesthetized with isoflurane $(2.5 \%$ for induction, $1.0 \%$ for maintenance). The mice were then intubated using PE 90 tubing at the supine position on a heating pad for mechanical ventilation. The aortic arch was constricted with a 5-0 silk suture tied around a blunt 27gauge blunt needle. The needle was removed after constriction. Sham-operated mice received similar surgical treatment with the omission of ligation. Three days after TAC or sham operation, the mice were further separated into two groups: the $B B G$ group which received P2X7R antagonist BBG (i.p. $30 \mathrm{mg} / \mathrm{kg}$ body weight in $0.9 \%$ saline $10 \mathrm{ml} / \mathrm{kg}$ ) (Sigma) three times per week and the saline group which received saline (i.p. $0.9 \% 10 \mathrm{ml} / \mathrm{kg}$ ). Four weeks after surgery, echocardiography was performed on all mice to assess cardiac function. At the end of experiment, the mice were sacrifice, and the hearts were excised and then snap-frozen in liquid nitrogen at $-80^{\circ} \mathrm{C}$ for further analysis.

2.2. Echocardiography. An echocardiography machine (iE33, Philips) fitted with a $35 \mathrm{MHz}$ transducer was employed to evaluate cardiac remolding in mice four weeks following TAC operation. An experienced operator who was blinded to the treatment groups performed and analyzed echocardiography results. Briefly, the mice were exposed to isoflurane to anesthetize them and placed in a supine position on a heating pad. Images were captured in the short axis of the left ventricle to calculate internal wall dimensions during systole and diastole. From M-mode images, the thick- ness and dimensions of the left ventricle (LV) chamber were obtained. LV systolic function was determined by calculating ejection fraction (EF) and fractional shortening (FS).

\subsection{Histological Analyses (Trichrome Staining, Sirius Red} Staining, Scar Size Measurement, and Immunohistochemistry). After echocardiographic evaluation, freshly isolated hearts were fixed with paraformaldehyde (4\%), embedded in paraffin, and transversally sectioned (thickness, 4-5 $\mu \mathrm{m}$ ). For immunohistochemistry (IHC) staining, tissues were incubated with antiperiostin (Abcam, 1:200), and the staining was performed as per the methods described previously [18]. Masson's trichrome and Sirius red staining (Sigma) were performed following the manufacturer's instructions. Staining crosssection heart tissue images were recorded using a brightfield microscope (Leica). To evaluate the degree of cardiac fibrosis, the National Institutes of Health (NIH) ImageJ software was used to analyze images by comparing blue- or redstained area (collagen) with the total area (10 sections were randomly chosen per heart, $n=6$ hearts per group).

2.4. Cell Culture and Pharmaceutical Treatments. Neonatal rat cardiac fibroblasts were isolated from the hearts of decapitated Sprague-Dawley rats (age, 0-3 days) according to the methods described previously [19]. The CFs were grown in a culture flask with DMEM mixed with 10\% FBS, and $100 \mathrm{U} / \mathrm{ml}$ of both streptomycin and penicillin. CFs at the second passaging were added into 6 -well plates and cultivated to reach $70-80 \%$ confluence. The NRCFs were incubated for 4-6 hours with serum-free DMEM and then later treated with TGF- $\beta 1$ (a known stimulator of NRCFs, $10 \mathrm{ng} / \mathrm{ml}$, Sino Biological Inc.) for $48 \mathrm{~h}$ to induce fibroblasts activation and fibrosis. A subset of cells was treated with BBG (100 nM) after $2 \mathrm{~h}$ exposure to TGF- $\beta 1$ or treated with BzATP $(100 \mu \mathrm{M})$ for $1 \mathrm{~h}$ at the end of stimulation.

2.5. Cell Transfection. Cells grown to $50-60 \%$ confluence were put in 6-well plates. They were transfected using transfection reagent riboFECT ${ }^{\mathrm{TM}} \mathrm{CP}$ ( RiboBio $^{\mathrm{TM}}$, China). siRNAs targeting P2X7R (siP2X7R) were transfected into NRCFs for 24 hours and then treated with TGF- $\beta 1$ for 48 hours. Individual siRNAs (100 nM, RiboBio ${ }^{\mathrm{TM}}$, China), riboFECT ${ }^{\mathrm{TM}} \mathrm{CP}$ reagent and buffer, and DMEM were combined and then incubated for 15 minutes at room temperature. The product code numbers of each siRNA are shown in Table 1.

2.6. Immunofluorescence Methodology. Immunofluorescence staining on cells was performed as described previously [19]. The primary antibody for incubation was anti- $\alpha$-SMA (Abcam, 1:200) or P2X7R (Alomone Labs, 1:200). Images were obtained using confocal microscopy and analyzed with the ImageJ software (NIH, USA).

2.7. Evaluation of Gene Expression. The real-time-PCR assay was performed to examine gene expression as per the methods described previously [18]. Briefly, total RNA was isolated from the mice hearts or cultured NRCFs using TRIzol (Invitrogen, USA). Next, the RNA was used as a template to synthesize cDNA with a reverse transcription (RT) kit (Toyobo, Japan). The qPCR was conducted using the SYBR 
TABLE 1: Sequences of siRNA to P2X7R.

\begin{tabular}{lc}
\hline Target & Sequences of SiRNA \\
\hline siP2X7R-1 & CCACAACUAUACUACGAGA \\
siP2X7R-2 & GAGGAAUCAUGGGCAUUGA \\
siP2X7R-3 & CGCUGUCAACCCAAAUACA \\
siRNA-NC & Provided by RiboBio ${ }^{\text {TM }}$ \\
\hline
\end{tabular}

TABLE 2: Rat RT-qPCR primer sequences.

\begin{tabular}{|c|c|}
\hline Target mRNA & Sequence $\left(5^{\prime} \longrightarrow 3^{\prime}\right)$ \\
\hline \multirow{2}{*}{ CTGF } & Forward: CCCTGACCCAACTATGATGC \\
\hline & Reverse: CCTTACTCCCTGGCTTTACG \\
\hline \multirow{2}{*}{ GAPHD } & Forward: AGTGCCAGCCTCGTCTCATA \\
\hline & Reverse: GATGGTGATGGGTTTCCCGT \\
\hline \multirow{2}{*}{ P2X7R } & Forward: CGAAGTTAGTACACGGCATCTT \\
\hline & Reverse: CTTGGCCTTCTGACTTGAGATAA \\
\hline \multirow{2}{*}{ Periostin } & Forward: ACGTCCTGGTGAAGTTGGTC \\
\hline & Reverse: GGTGGATGACACCATTCTTC3 \\
\hline \multirow{2}{*}{ Col-1 } & Forward: ACGTCCTGGTGAAGTTGGTC \\
\hline & Reverse: TCCAGCAATACCCTGAGGTC \\
\hline \multirow{2}{*}{ TGF- $\beta$} & Forward: TGAGTGGCTGTCTTTTGACG \\
\hline & Reverse: ACTGAAGCGAAAGCCCTGTA \\
\hline \multirow{2}{*}{$\alpha$-SMA } & Forward: CCGAGATCTCACCGACTACC \\
\hline & Reverse: ATGCCACAGGATTCCATACCC \\
\hline
\end{tabular}

Green Supermix kit (Bio-Rad, USA) on the BIORADCFX96 ${ }^{\mathrm{TM}}$ Real-Time PCR Detection System and GAPDH served as the reference gene. The sequences of the primers used are displayed in Tables 2 and 3 . The $2^{-\Delta \Delta \mathrm{Ct}}$ threshold (Ct) method was used to calculate relative fold changes.

2.8. Western Blot Analysis. Mice tissues and cells were treated with (RIPA) lysis buffer to extract total proteins according to the protocol provided by the manufacturer. Subsequently, the BCA protein assay kit (Pierce, USA) was employed for protein quantification. An equivalent amount of cell or tissue lysates $(25 \mu \mathrm{g})$ were denatured and separated through SDSPAGE (10\%) and then transferred to $0.45 \mu \mathrm{m}$ PVDF membrane (Millipore, USA) using a Mini-PROTEAN III system (Bio-Rad, USA) [20]. Next, the membranes were blocked for 2 hours with 5\% skimmed milk in TBST (tris-buffered saline) and Tween 20 solutions. After that, the membranes were incubated overnight $\left(4^{\circ} \mathrm{C}\right)$ alongside the following primary antibodies: connective tissue growth factor (CTGF) (Abcam, 1:1000), NLRP3 (HuaBio, $1: 1000)$, transforming growth factor $\beta$ (TGF- $\beta$ ) (Abcam, 1:1000), periostin (Abcam, 1:1000), P2X7R (Affinity Biosciences, $1: 1000$ ), $\alpha$ smooth muscle actin ( $\alpha$-SMA) (Abcam, $1: 1000)$, IL- $1 \beta$ (HuaBio, $1: 1000$ ), caspase-1, and GAPDH (Cell Signaling Technology, 1:1000). The membranes were treated with horseradish peroxide-conjugated anti-rabbit or goat antimouse secondary antibodies (Zsgb Bio, 1:2000) for 2 hours at room temperature. The blots were visualized with enhanced chemiluminescence (ECL) substrate kit (Thermo, USA).
TABLE 3: Mice RT-qPCR primer sequences.

\begin{tabular}{ll}
\hline Target mRNA & Sequence $\left(5^{\prime} \longrightarrow 3^{\prime}\right)$ \\
\hline CTGF & Forward: GGACACCTAAAATCGCCAAGC \\
& Reverse: ACTTAGCCCTGTATGTCTTCACA \\
GAPHD & Forward: AATGGATTTGGACGCATTGGT \\
& Reverse: TTTGCACTGGTACGTGTTGAT \\
P2X7R & Forward: CAGCGGAAAGAGCCTGTTATC \\
& Reverse: TGGCCTTCTGACTTGACATAGTT \\
Periostin & Forward: TGGTATCAAGGTGCTATCTGCG \\
& Reverse: AATGCCCAGCGTGCCATAA \\
Col-1 & Forward: TAAGGGTCCCCAATGGTGAGA \\
& Reverse: GGGTCCCTCGACTCCTACAT \\
TGF- $\beta$ & Forward: CTTCAATACGTCAGACATTCGGG \\
& Reverse: GTAACGCCAGGAATTGTTGCTA \\
$\alpha$-SMA & Forward: GGACGTACAACTGGTATTGTGC \\
& Reverse: TCGGCAGTAGTCACGAAGGA \\
Pannexin1 & Forward: GCTGCACAAGTTCTTCCCCTA \\
& Reverse: CGCGGTTGTAGACTTTGTCAAG \\
\hline
\end{tabular}

Finally, the ImageJ software (NIH, USA) was used to evaluate the protein band intensities.

2.9. Assessment of Cell Proliferation with EdU Assay. The proliferative activity of cells was determined using 5-ethynyl-2-deoxyuridine (EdU) staining (Click-iT ${ }^{\mathrm{TM}}$ EdU Alexa Fluor $^{\mathrm{TM}} 555$ Imaging Kit, Thermo Fisher Scientific). Cellular organelles were stained by incubating cells with $50 \mu \mathrm{M}$ EdU for 1 hour, and the nuclei were counterstained with DAPI for 30 minutes. The percentage of EdU-positive cells was defined as the cell proliferation rate. And was calculated as the number of EdU-positive cells normalized to the number of DAPI-stained cells observed under a fluorescent microscope (Olympus). All assays were performed more than thrice.

2.10. Data Analysis. Data were analyzed using the SPSS 22.0 software and presented as mean \pm SEM. For unpaired data, differences between groups were compared with Student's $t$-test or one-way analysis of variance (ANOVA). $P$ value $<0.05$ was taken as significant.

\section{Results}

3.1. Role of P2X7R in Pressure Overload-Induced Cardiac Fibrosis and CF Activation. Firstly, we explored the P2X receptor subtype(s) involved in TAC-induced cardiac fibrosis. Cardiac fibrosis following TAC was assessed using heart size, echocardiography indicators, HE, Masson's trichrome, and Sirius red staining. Figures $1(\mathrm{a})-1(\mathrm{c})$ show that TAC mice had higher heart weight relative to body weight $(\mathrm{HW} / \mathrm{BW})$ ratios, area of fibrosis and cross-sectional, left ventricular end-diastolic posterior wall thickness (LVPWd), LV internal diameter in diastole (LVIDd), and interventricular septal thickness (IVSd) but reduced fractional shortening (FS\%) and ejection fraction (LVEF\%) compared with the 


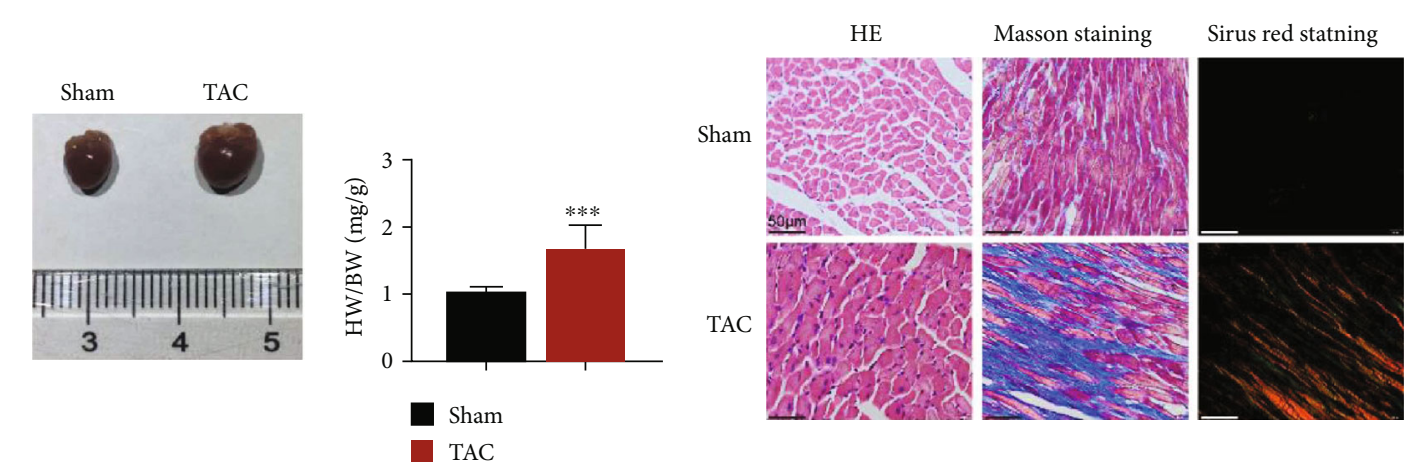

(a)

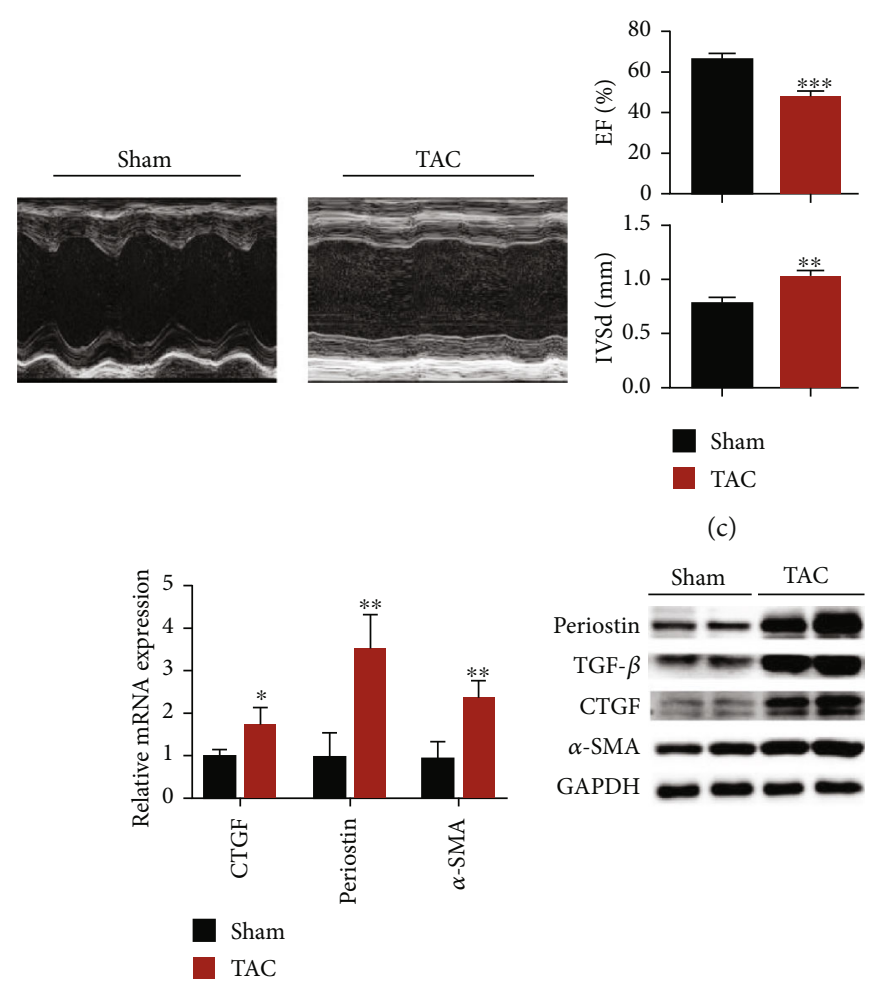

(d)

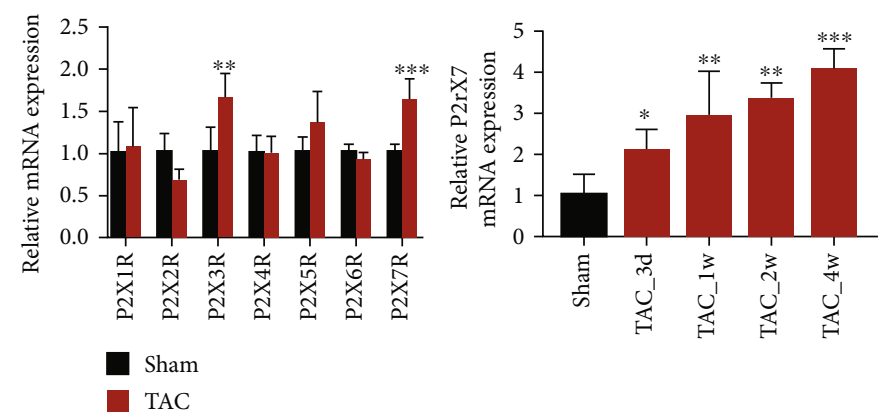

(e) (b)

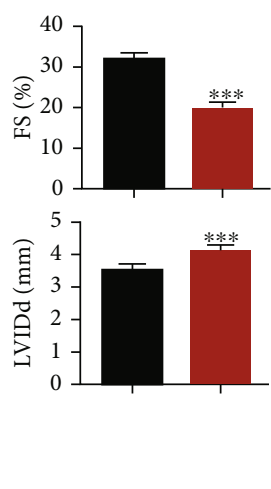

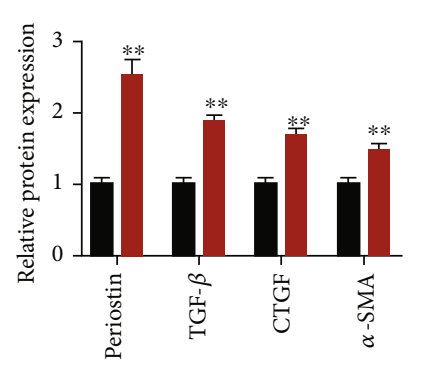

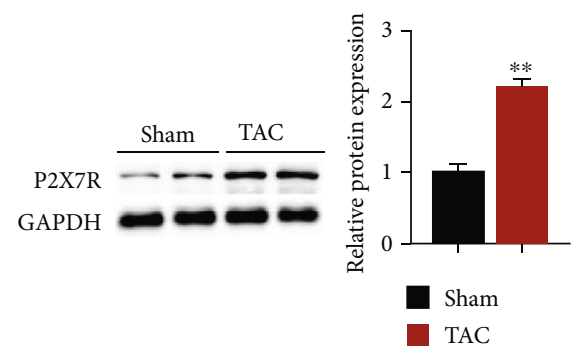

(f)

Figure 1: P2X7R was upregulated in the mice heart after TAC surgery. (a) Cardiac images and HW/BW ( $n=8)$. (b) HE-, Sirius Red-, and Masson's trichrome-stained sections of the hearts following four weeks of TAC treatment $(n=6)$. (c) Representative images of an echocardiographic assessment of mice after TAC (4 weeks). Cardiac function indicators measured by echocardiography (LVEF (\%), FS (\%), LVPWd, IVSd, and LVIDd) in TAC- and sham-operated mice. (d) Expression of CTGF, periostin, and $\alpha$-SMA mRNAs and proteins in heart cells $(n=6)$. (e) Gene expression of P2X receptors in TAC- and sham-operated mice $(n=6)$ at 4 weeks and gene expression of P2X7R in heart cells at 3 days, 1 week, 2 weeks, and 4 weeks after TAC- or sham-operated treatment $(n=6)$. (f) Expression of P2X7R protein in mouse heart cells $(n=6)$. Results are presented as means \pm standard error of the mean. ${ }^{*}$ indicates $P<0.05,{ }^{* *}$ indicates $P<0.01$, and ${ }^{* * *}$ indicates $P<0.001$ versus sham. 

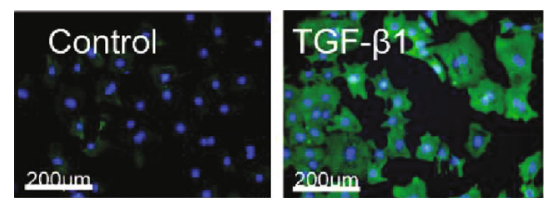

(a)

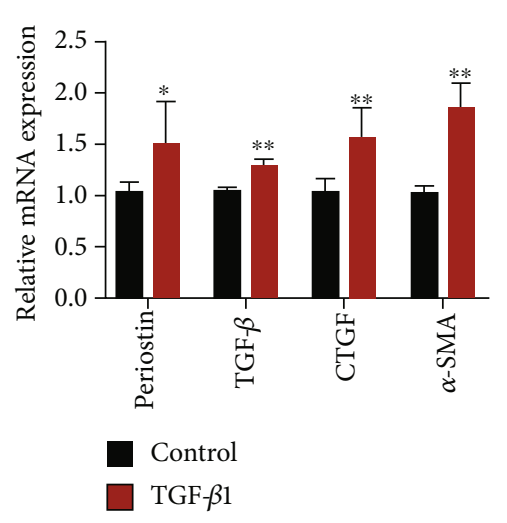

(c)

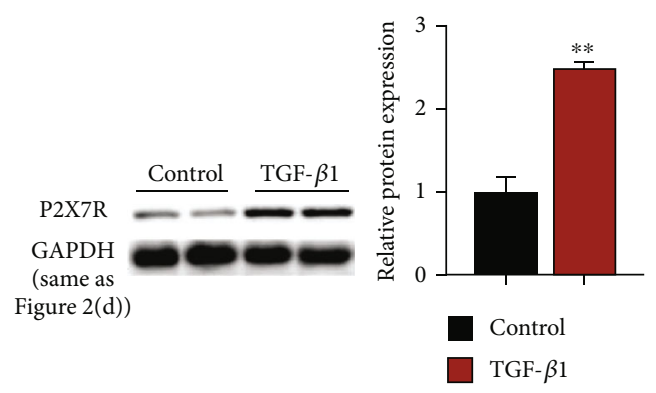

(e)

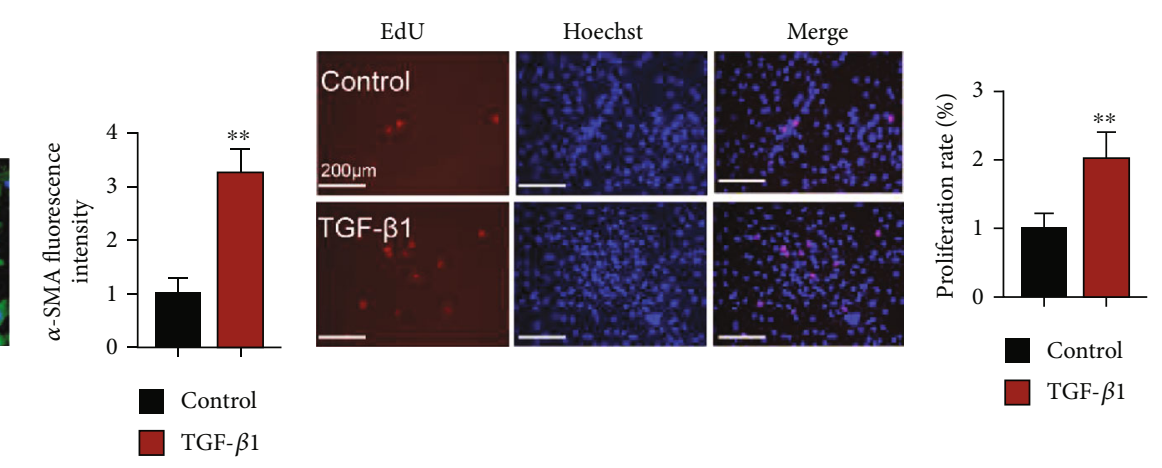

(b)
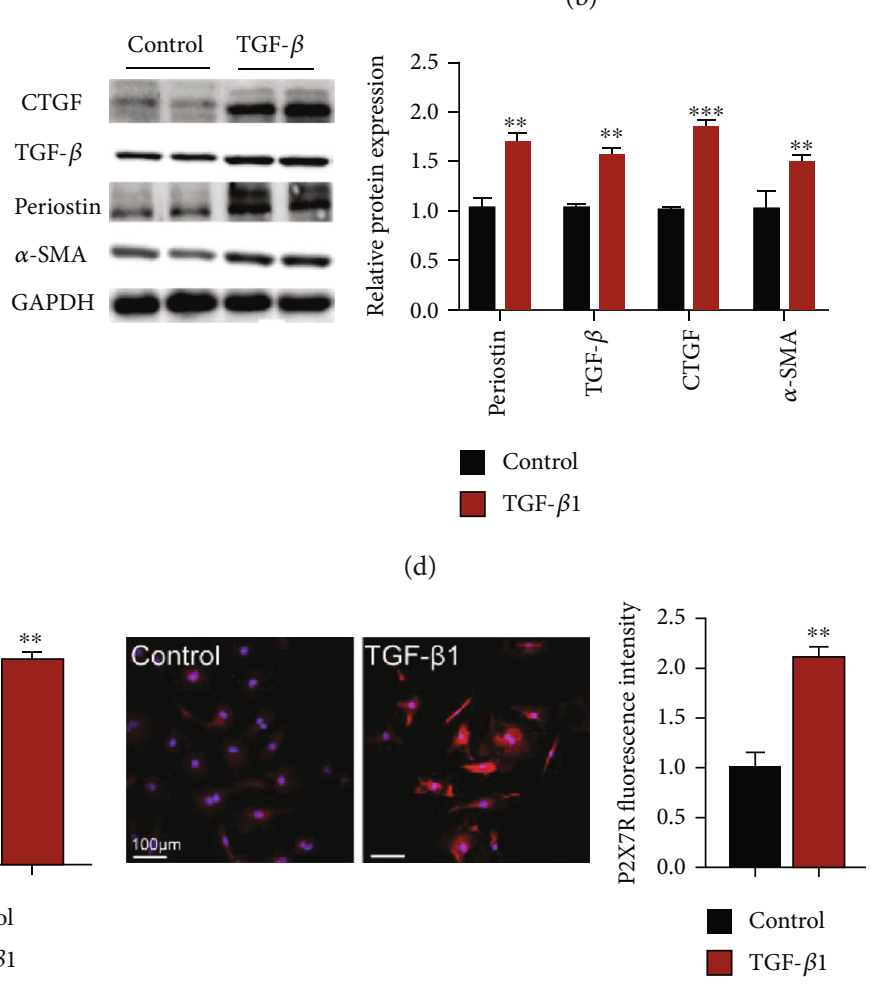

(f)
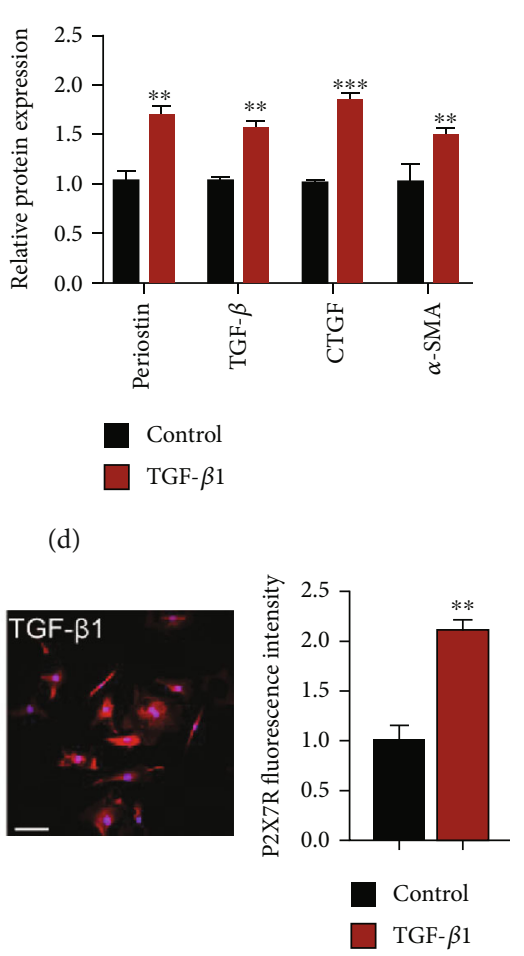

(d)

Figure 2: TGF- $\beta 1$-treatment upregulated P2X7R in CFs. (a) Immunofluorescence images showing $\alpha$-SMA expression in CFs ( $\alpha$-SMA (green) and nuclei DAPI (blue), $n=300$ ). (b) Rate of cell proliferation (EdU-positive cells (red) and nuclei DAPI (blue), $n=120)$. (c) Expression of TGF- $\beta, \alpha$-SMA, CTGF, and periostin mRNAs $(n=3)$. (d) Expression of CTGF, $\alpha$-SMA, periostin, and TGF- $\beta$ proteins $(n=3)$. (e) Western blot results showing P2X7R expression in CFs $(n=3)$. (f) Immunofluorescence images showing P2RX7 expression in CFs (P2X7R (red) and nuclei DAPI (blue), $n=60$ ). Results are presented as means \pm standard error of the mean; ${ }^{*}$ indicates $P<0.05$, ${ }^{* *}$ indicates $P<0.01$, and *** indicates $P<0.001$ versus control.

sham group. In addition, mRNA expressions of CTGF, periostin, and $\alpha$-SMA were about $1.7,3.5$, and 2.3 times higher, respectively, in TAC mice relative to the sham group mice (Figure $1(\mathrm{~d})$ ). The periostin, TGF- $\beta, \alpha$-SMA, and CTGF protein levels were also higher by 2.5-, 1.8-, 1.7-, and 1.5-fold, respectively, in TAC mice compared to the sham group mice (Figure 1(d)).

RT-PCR analysis was performed to detect expression of all $\mathrm{P} 2 \mathrm{X}$ receptors mRNAs in the mouse hearts. Expressions of P2X3R and P2X7R mRNAs were higher in TAC relative to the sham mice hearts (Figure 1(e)). Among them, P2X7R began to increase three days after surgery, reaching the highest level in the 4th week (Figure 1(e)). Similarly, in the TAC group, the protein level of P2X7R was higher approximately by 2.1 -fold in comparison to the sham group (Figure $1(\mathrm{f})$ ).

Subsequently, we examined the expression of P2X7R in neonatal rat cardiac fibroblasts following TGF- $\beta 1(10 \mathrm{ng} / \mathrm{ml}$ for $48 \mathrm{~h}$ ) treatment. The expressions of profibrosis markers' mRNAs, that is, periostin, CTGF, TGF- $\beta$, and $\alpha$-SMA, were higher by $1.5,1.3,1.5$, and 1.8 times, respectively, in CFs treated with TGF- $\beta 1$ than in control group (Figure 2(c)). Also, the expressions of CTGF, TGF- $\beta$, periostin, and $\alpha$ SMA proteins were higher by $1.7,1.5,1.8$, and 1.5 times, respectively, in fibroblasts treated with TGF- $\beta 1$ in comparison to the control group (Figure 2(d)). Moreover, $\alpha$-SMA and P2X7R staining intensities were higher by 3 -fold and 
2.1-fold, respectively, in CFs treated with TGF- $\beta 1$ than in control group (Figures 2(a) and 2(f)). The number of EdU-positive cells were 2-fold higher (Figure 2(b)) in NRCFs treated with TGF- $\beta 1$ than control NRCFs. Meanwhile, the protein expression of P2X7R in fibrotic CFs was higher by almost 2.4 times relative to the untreated controls (Figure 2(e)). These findings imply that P2X7R expression elevated following pathological activation of NRCFs.

3.2. Suppression of $P 2 X 7 R$ s Protects CFs from TGF- $\beta 1$ Induced Activation. To investigate whether P2X7Rs participated in TGF- $\beta 1$-induced CF activation, siP2X7R silencing vector, and P2X7R inhibitor BBG were employed. Results showed that transfection with $\mathrm{siP} 2 \mathrm{X} 7 \mathrm{R}$ reduced mRNA expression levels of P2X7R by nearly 65\% (Figure 3(a)), and that of CTGF (by 26\%), TGF- $\beta$ (by 28\%), Col-1 (by $48 \%$ ), and $\alpha$-SMA (by $27 \%$ ) compared with the control group cells (Figure 3(b)). At the protein level, CTGF, periostin, $\alpha$-SMA, and P2X7R decreased by $27 \%, 28 \%, 31 \%$, and $24 \%$, respectively, relative to the control cells (Figure $3(\mathrm{c})$ ). The relative fluorescence intensity of $\alpha$-SMA in the CFs transfected with siP2X7R decreased by approximately 46\% (Figure 3(e)). Moreover, the number EdU-positive cells in the group transfected with siP2X7R was fewer by nearly 38\% compared to those of the control group (Figure $3(\mathrm{f})$ ). The expression of P2X7R in cells treated with BBG after TGF- $\beta 1$ stimulation was lower than that of cells treated with TGF- $\beta 1$ alone (Figure 3(h)). As expected, treatment of cells with BBG after TGF- $\beta 1$ stimulation reduced mRNA and protein levels of fibrosis markers (Figures 3(g) and 3(i)). In contrast, treatment with the P2X7R agonist, BzATP, increased the activation of CFs, as evidenced by 1.2-, 1.3-, 1.2-, and 1.2-fold elevation in periostin, CTGF, $\alpha$-SMA, and TGF- $\beta$ protein expression (Figure $3(\mathrm{j})$ ). These data sets demonstrate that downregulating P2X7R ameliorates CF activation.

3.3. Downregulation of P2X7Rs Suppresses NLRP3/IL-1 $\beta$ Expression in TGF- $\beta 1$-Induced CF Activation. Herein, we assessed the expression of three arms of NLRP3/IL- $1 \beta$ signaling pathways, including NLRP3, caspase- 1 , and IL- $1 \beta$ to elucidate whether NLRP3/IL-1 $\beta$ pathway was activated following TGF- $\beta 1$ stimulation. Figure $3(\mathrm{~d})$ shows that these proteins were upregulated in the TGF- $\beta 1$ group.

Next, we determined whether P2X7Rs regulated the NLRP3/IL-1 $\beta$ pathway. P2X7R knockdown in CFs treated with TGF- $\beta 1$ led to lower levels of NLRP3 (20\%), IL-1 $\beta$ $(21 \%)$, and caspase-1 (15\%) compared with their corresponding levels in the siNC+TGF- $\beta 1$ group (Figure $3(\mathrm{~d})$ ).

Moreover, CFs cotreated with TGF- $\beta 1$ and a P2X7Rspecific inhibitor, and BBG had lower NLRP3, IL- $1 \beta$, and caspase-1 levels in BBG+TGF- $\beta 1$ than fibroblasts treated with TGF- $\beta 1$ alone (Figure 3(i)).

3.4. Inhibition of P2X7Rs Suppresses Pressure OverloadInduced Cardiac Remodeling. Further experiments were performed to tests whether P2X7Rs were involved in pressure overload-induced cardiac fibrosis. Results showed that mice of the TAC+BBG group administered with BBG 2 days after TAC procedure showed a significantly lower $\mathrm{HW} / \mathrm{BW}$ ratio, area of fibrosis, and number of periostin-positive cells than the mice in the TAC+saline group (Figures 4(a)-4(d)). In addition, treatment with BBG in the TAC group significantly improved EF (\%), FS (\%), LVIDd, and LVPWd compared with the TAC+saline treatment group (Figure 4(e)). Furthermore, the treatment with BBG decreased the protein expressions of CTGF, periostin, and $\alpha$-SMA in the TAC group (Figure 4(f)).

We also examined whether pressure overload altered NLRP3/IL- $1 \beta$ signaling pathways in TAC mice. In the TAC +saline group, the NLRP3, IL- $1 \beta$, and caspase- 1 protein levels were higher by 2.2, 2.0, and 1.5 times, respectively, in comparison to the sham+saline group. In contrast, NLRP3, IL- $1 \beta$, and caspase- 1 levels were significantly lower by $11 \%$, $25 \%$, and $20 \%$, respectively, in the $\mathrm{TAC}+\mathrm{BBG}$ group relative to the TAC+saline group (Figure $4(\mathrm{f})$ ).

These data suggest that $\mathrm{P} 2 \mathrm{X} 7 \mathrm{R}$ inhibition ameliorates cardiac dysfunction and cardiac fibrosis induced by pressure overload.

\section{Discussion}

In this study, we examined whether P2X7R participates in cardiac fibrosis induced by pressure overload. The main results are shown in Figure 5.

Studies show that cellular activation, mechanical stimulation, or injuries induce ATP release into extracellular spaces, contributing to inflammatory reactions and fibrosis in injured heart cells $[6,21,22]$. Activation of the $\mathrm{P} 2 \mathrm{X}$ receptor by nucleotides modulates cell migration, proliferation, inflammation, cytokines release, and necrosis [23]. P2X7R is an ATP ligand-gated cation channel that responds to extracellular ATP levels and plays a role in tissue fibrosis. In a silica-induced lung fibrosis mouse model, knockout of P2X7R in mice reduced lung fibrosis and inflammation. In vitro studies have shown that inhibition of the P2X7R prevented inflammatory responses in silica-treated fibroblasts and alveolar macrophages [24]. Tung et al. investigated the role of P2X7R in liver cirrhosis. They found that inhibition of P2X7R reduced hepatic inflammatory cytokines and suppressed TGF- $\beta$ signaling pathway [25]. Similarly, P2X7R regulates heart responses to injury. Therefore, it plays a role in several heart conditions such as hypertension, acute myocardial infarction (AMI), and experimental autoimmune myocarditis (EAM) $[14,16,26,27]$. However, the precise role of P2X7R in cardiac fibrosis is not known. Our results demonstrated that hearts from TAC mice exhibited elevated P2X7R expression. Although P2X7R is expressed in the human heart, it is clear whether it is expressed in cardiomyocytes and CFs [9]. Herein, the P2X7R protein was not detected in ventricular cardiomyocytes, but it was detected in endothelial cells and CFs [13, 28-30]. Herein, similar to in vivo findings, in vitro assays revealed that $\mathrm{P} 2 \mathrm{X} 7 \mathrm{R}$ was highly expressed in activated CFs.

The P2X7R antagonist, BBG, has been reported to exert therapeutic effects against lung and liver damage by preventing oxidative damage and inflammation. In a carrageenan(CAR-) induced pleurisy mouse model, BBG decreased the assembly of the NRLP3/ASC/caspase-1 complex and iNOS, 


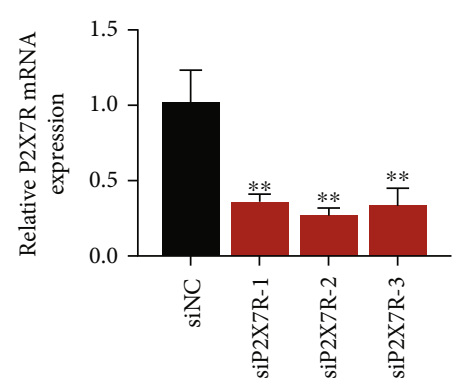

(a)

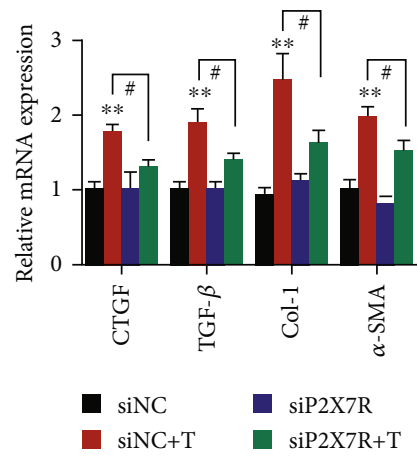

(b)
siNC siNC+T siP2X7R siP2X7R+T

CTGF

NLRP3

Periostin $=-\infty-\infty=0$

P2X7R

${ }^{\alpha-S M A}-7$

${ }_{\mathrm{ILL}-\beta}=$ ニニニニニニ

Casp-1

GAPDH

(c)

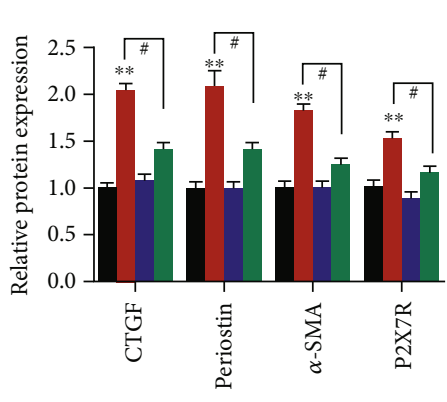

- siNC

aiNC+T

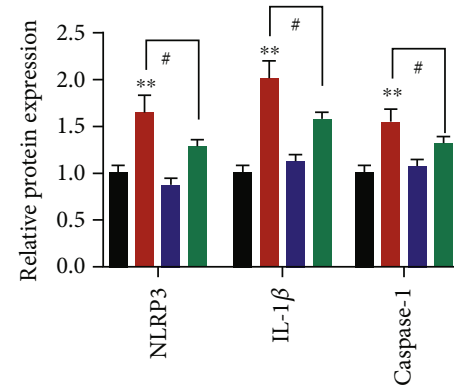

- $\operatorname{siP2X7R}$

siP2X7R+T

(d)
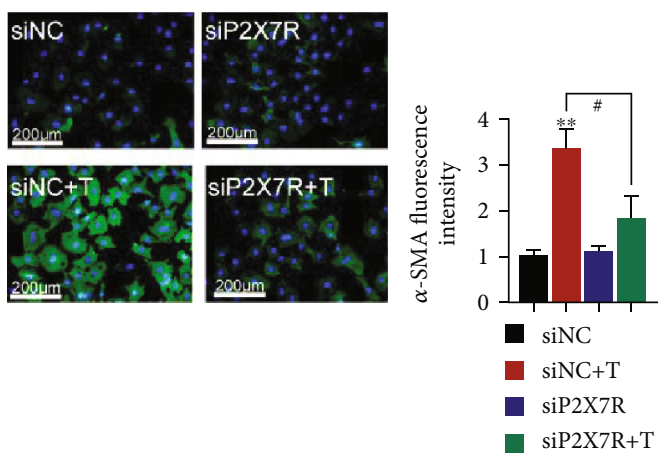

(e)
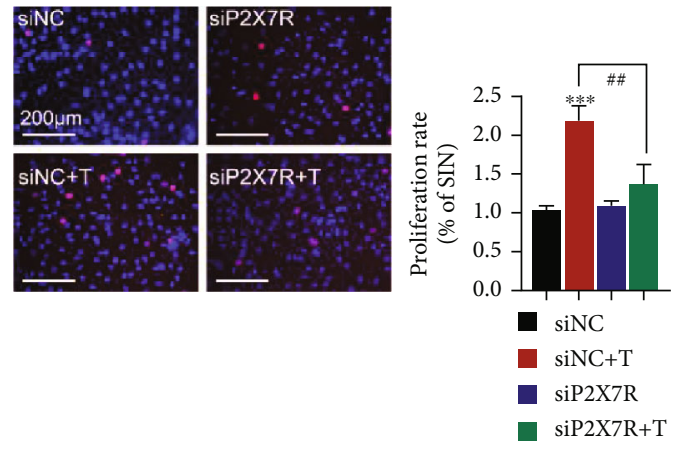

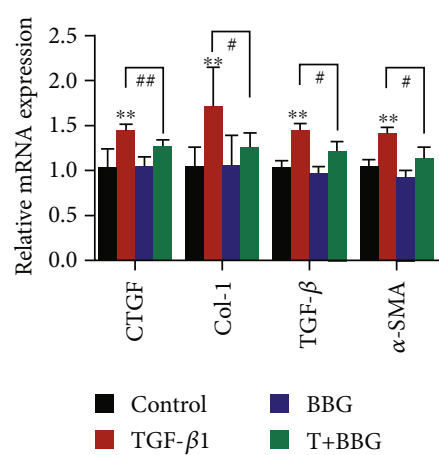

(g)
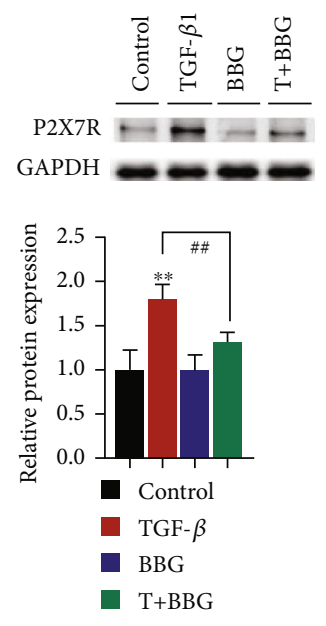

(h)

Figure 3: Continued. 

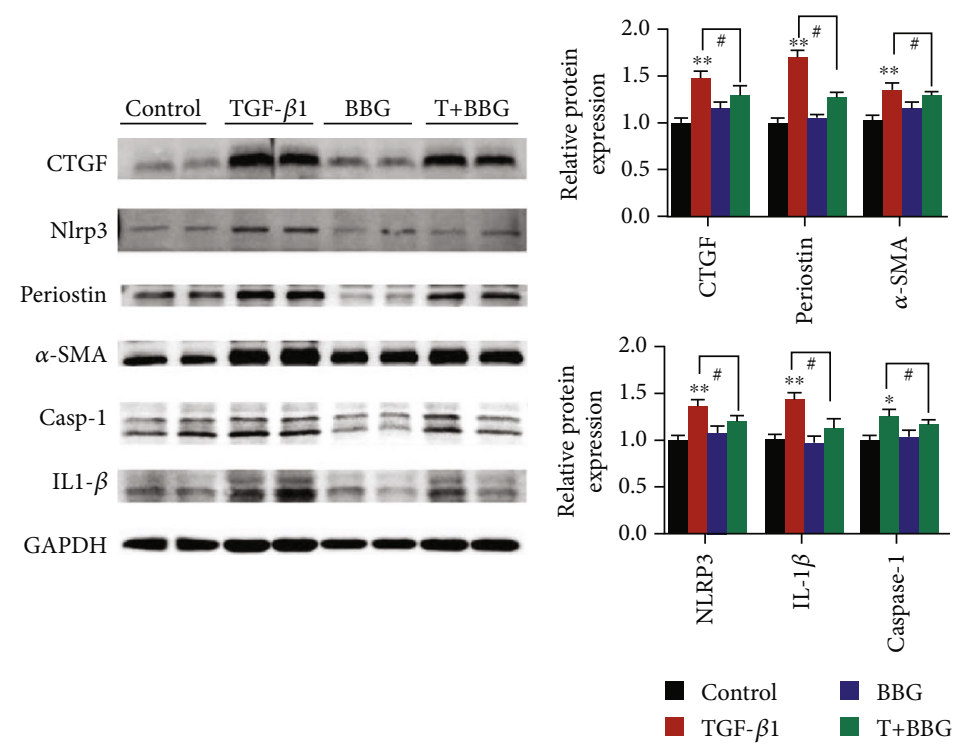

(i)
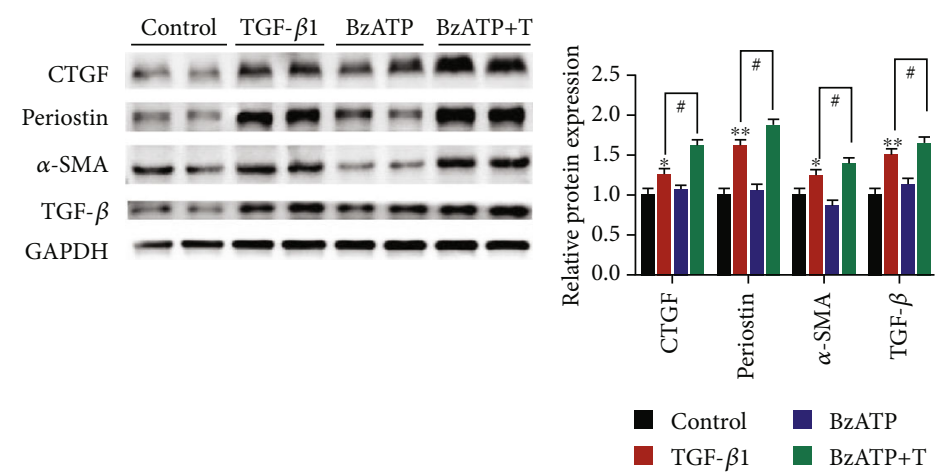

(j)

Figure 3: Knockdown of P2X7R alleviates the effects of TGF- $\beta 1$ on CF activation. (a) Expression of P2X7R mRNA after siP2X7R transfection $(n=6)$. (b) Expression of collagen I, CTGF, $\alpha$-SMA, and TGF- $\beta$ mRNAs after transfection of CFs with siP2X7R $(n=6)$. (c, d) Expression of periostin, $\alpha$-SMA, CTGF, P2X7R, NLRP3, IL-1 $\beta$, and caspase-1 proteins after transfection of CFs with siP2X7R ( $n=6)$. (e) Expression of $\alpha$ SMA in CFs by immunofluorescence staining ( $\alpha$-SMA (green) and nuclei DAPI (blue), $n=300$ ). (f) Changes in CF proliferation (EdUpositive cells (red) and nuclei DAPI (blue), $n=120$ ). (g) Expression of collagen I, CTGF, $\alpha$-SMA, and TGF- $\beta$ mRNAs after treatment of CFs with BBG $(n=6)$. (h) Expression of P2X7R protein after BBG treatment $(n=6)$. (i) Expression of periostin, $\alpha$-SMA, CTGF, NLRP3, IL- $1 \beta$, and caspase- 1 proteins after BBG treatment $(n=6)$. (j) Expression of periostin, $\alpha$-SMA, TGF- $\beta$ and CTGF proteins in activated CFs after BzATP treatment $(n=6)$. Results are presented as means \pm standard error of the mean; ${ }^{*}$ indicates $P<0.05,{ }^{* *}$ indicates $P<0.01$, and ${ }^{* * *}$ indicates $P<0.001$ versus control or siNC; ${ }^{\#}$ indicates $P<0.05$, ${ }^{\# \#}$ indicates $P<0.01$, and ${ }^{\# \# \#}$ indicates $P<0.001$.

nitrotyrosine, and poly-ADP-ribosyl polymerase expression [31]. In a mouse model of acetaminophen- (APAP-) induced hepatotoxicity, a combination of celastrol and BBG prevented the hepatic antioxidant consumption (decreased superoxide dismutase and glutathione) and hepatocellular injury [32]. Elsewhere, administration of BBG decreased superoxide dismutase (SOD), malondialdehyde (MDA), TNF- $\alpha$, and IL- $1 \beta$ concentration in pulmonary arterial hypertension (PAH) and ischemia-reperfusion- (IR-) induced lung models [33]. In our TAC mouse model, BBG affected several pathways controlled by P2X7R. We found that BBG partially attenuated the abnormal cardiac function caused by pressure overload. This outcome is consistent with that of P2X7R inhibitor, PPADS, both of which reduced cell death and cardiac fibrosis in mice with AMI [29]. Previous studies show that P2X7R was highly expressed in the cervical sympathetic ganglia in MI rats, but treatment with BBG or P2X7R siRNA restored P2X7R expression [34]. Consistently, we found that P2X7R mRNA and protein levels were elevated in CFs primed with TGF- $\beta 1$. This effect was attenuated by P2X7R gene silencing. Similarly, BBG prevented CF activation by inhibiting P2X7R. However, P2X7R activation with BzATP agonist aggravated the effect of TGF- $\beta 1$ on CFs. Thus, P2X7R participates in the pathogenesis of cardiac fibrosis and fibroblasts activation.

P2X7R is activated by high extracellular ATP [35]. Previous works suggest that ATP released from cardiomyocytes via pannexins initiates fibroblast proliferation and induces TGF- $\beta$ expression in rat fibroblasts $[36,37]$. We thus postulate that ATP released from cardiac cells accumulates in 

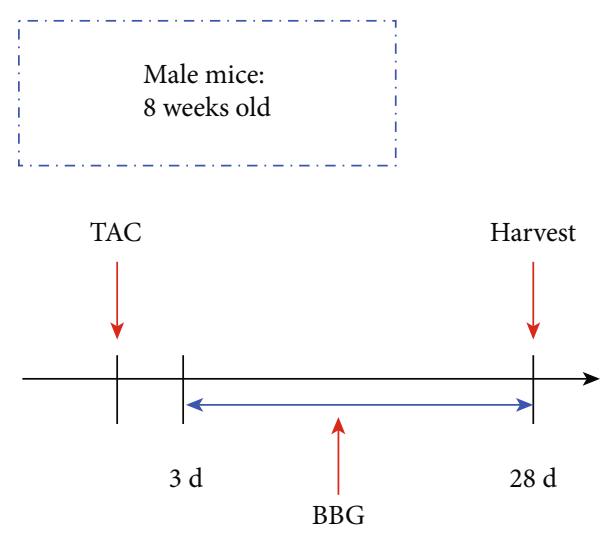

(a)
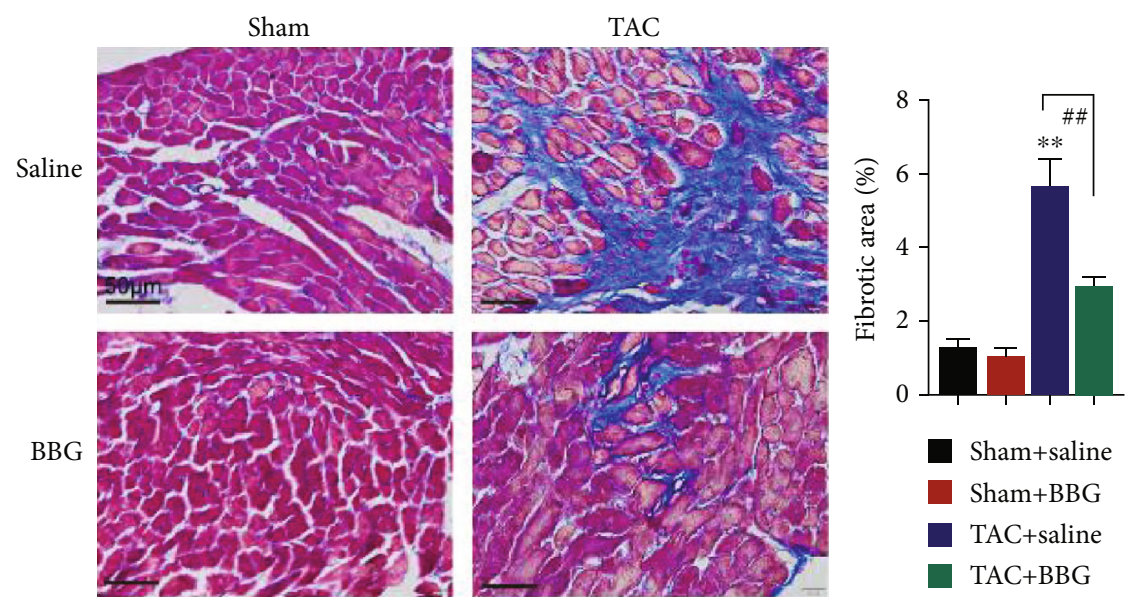

(c)

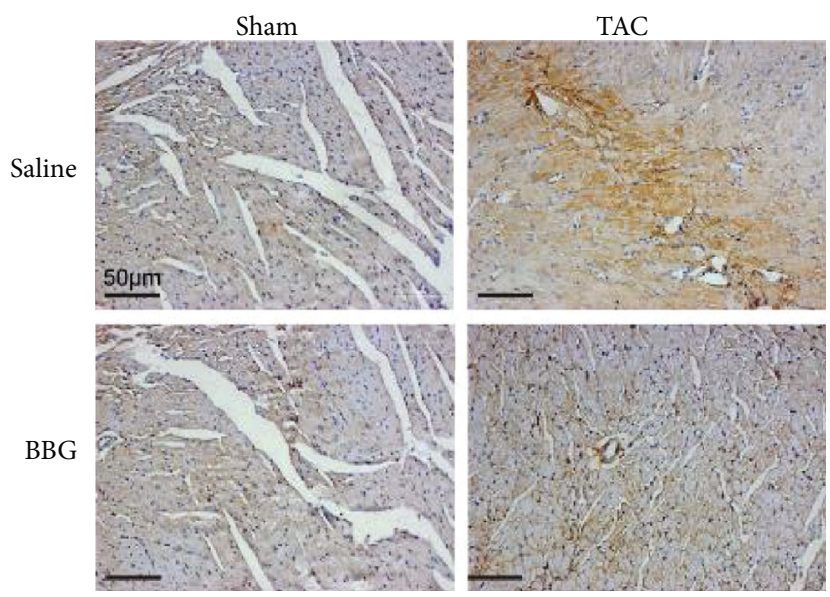

(d)

Figure 4: Continued. 

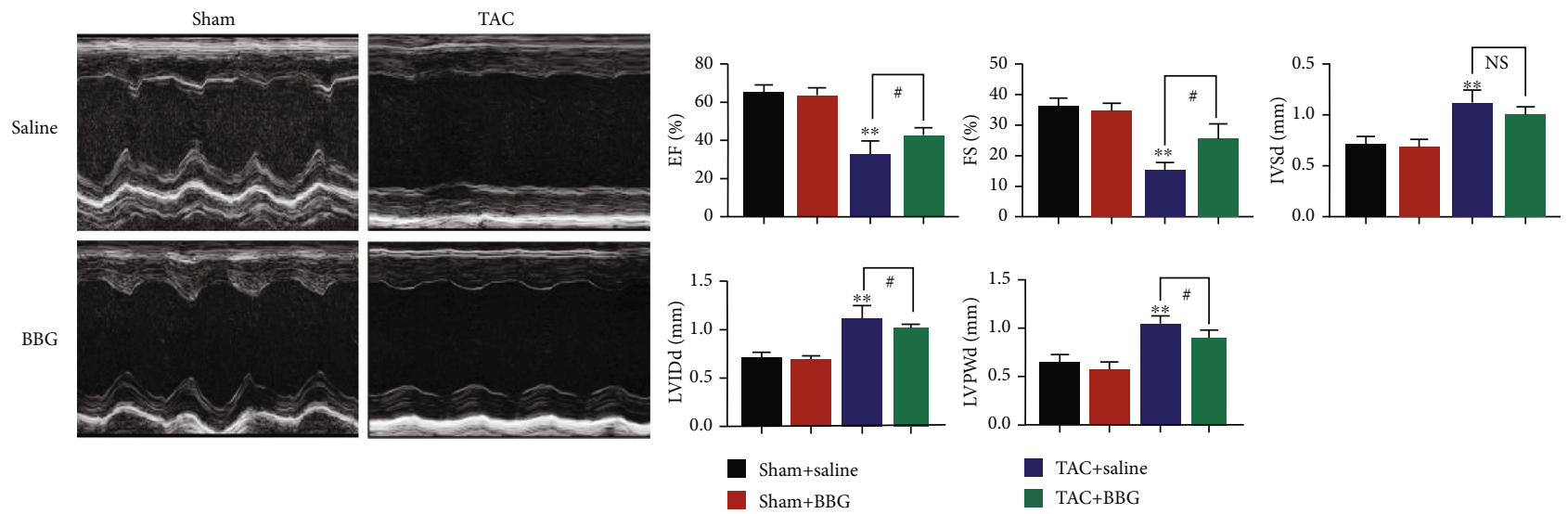

(e)
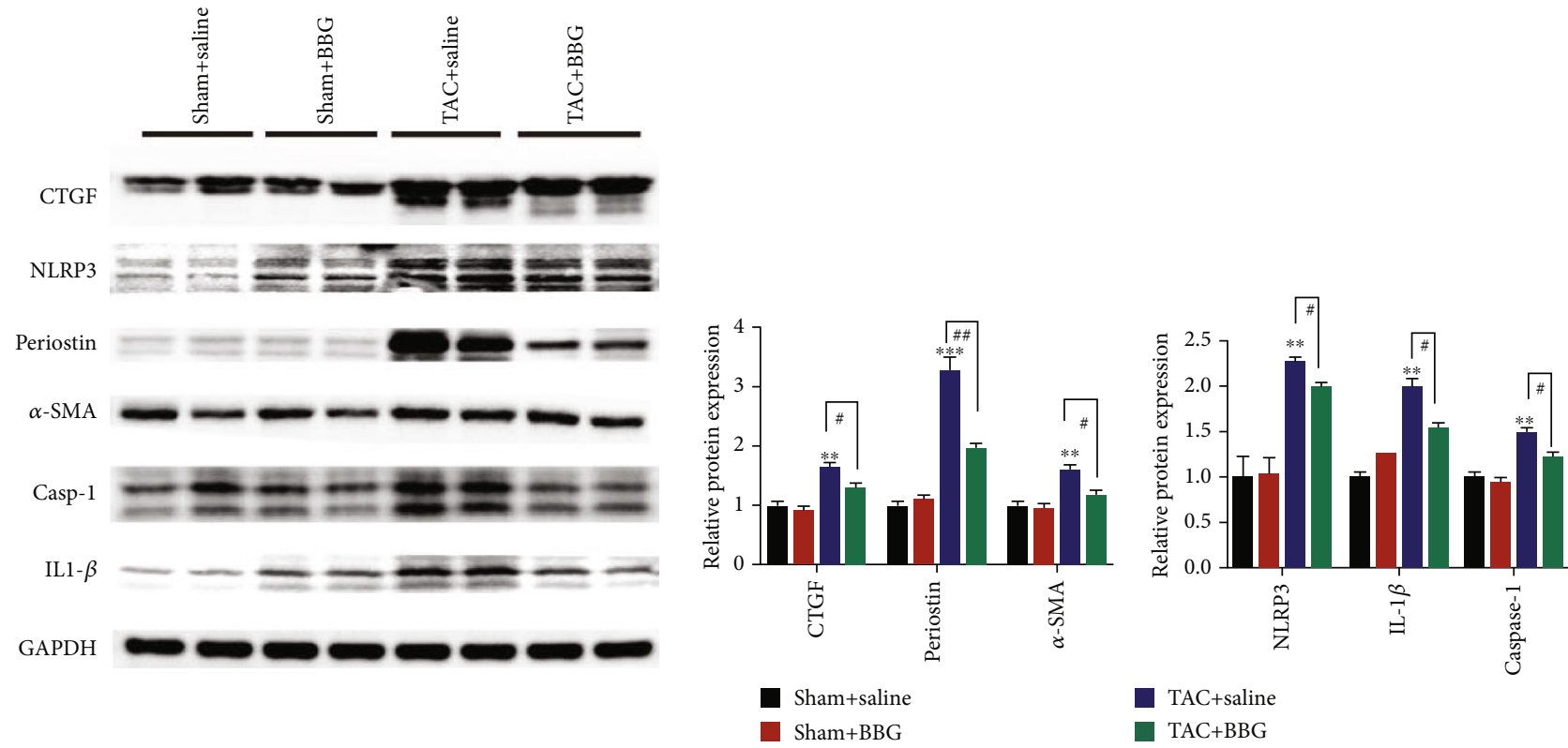

(f)

FIgure 4: Inhibition of P2X7Rs suppresses cardiac fibrosis induced by pressure overload. (a-d) Effects of BBG on TAC-triggered fibrosis (a, b), histological (c, d), echocardiographic analyses (e). (a) Schematic diagram outlining the in vivo experiments. Mice were treated with BBG every two days at three days after TAC. (b) HW/BW and cardiac imaging results $(n=6)$. (c) Masson's trichrome staining $(n=6)$. (d) Periostin protein levels in ventricular tissues (periostin (brown) and nucleus (blue). (e) Representative echocardiographic images of mice after TAC (4 weeks); the cardiac function indicators evaluated by echocardiography (LVEF (\%), FS (\%), LVPWd, IVSd, and LVIDd) in TAC- and sham-operated mice. (f) Expression of periostin, $\alpha$-SMA, CTGF, NLRP3, IL- $1 \beta$, and caspase- 1 proteins $(n=6)$. Results are presented as means \pm standard error of the mean; * indicates $P<0.05$, ${ }^{* *}$ indicates $P<0.01$, and ${ }^{* * *}$ indicates $P<0.001$ versus sham+saline. ${ }^{\#}$ indicates $P<0.05,{ }^{\# \#}$ indicates $P<0.01$, and ${ }^{\# \#}$ indicates $P<0.001$.

extracellular space through pannexins in mice subjected to TAC. This is evidenced by the upregulated expression of pannexin1 in TAC mice in this study (Figure S1). Studies have shown that TGF- $\beta 1$ activation promotes cardiac fibrosis by inducing the transformation of fibroblasts into myofibroblasts leading to excessive collagen generation [38]. Other studies have shown that ATP released from cardiomyocytes in response to mechanical stretching stimulated purinergic receptors in $\mathrm{CFs}$ in a paracrine manner [39]. We speculate that stretch-triggered ATP release in the context of TAC may activate P2X7R receptors expressed on $\mathrm{CFs}$, contributing to high collagen synthesis.
We also found that NLRP3/IL-1 $\beta$ signaling participates in P2X7R-mediated cardiac fibrosis. NLRP3/IL- $1 \beta$ is activated along with increased P2X7R expression. NLRP3 inflammasome regulates chronic sterile inflammation in response to intrinsic host signals released by injured cells. Activation of inflammasomes contributes to cleavage of pro-caspase- 1 to its active form which further cleaves inactive pro-IL- $1 \beta$ into IL- $1 \beta$. Activation of P2X7R is thus a vital step in the formation of NLRP3 inflammasome. In this way, P2X7R activation promotes cardiac dysfunction, cell death, and cardiac fibrosis $[13,40]$. In a calcineurin-transgenic(CNTg-) induced heart failure mice model, IL-1 receptor 


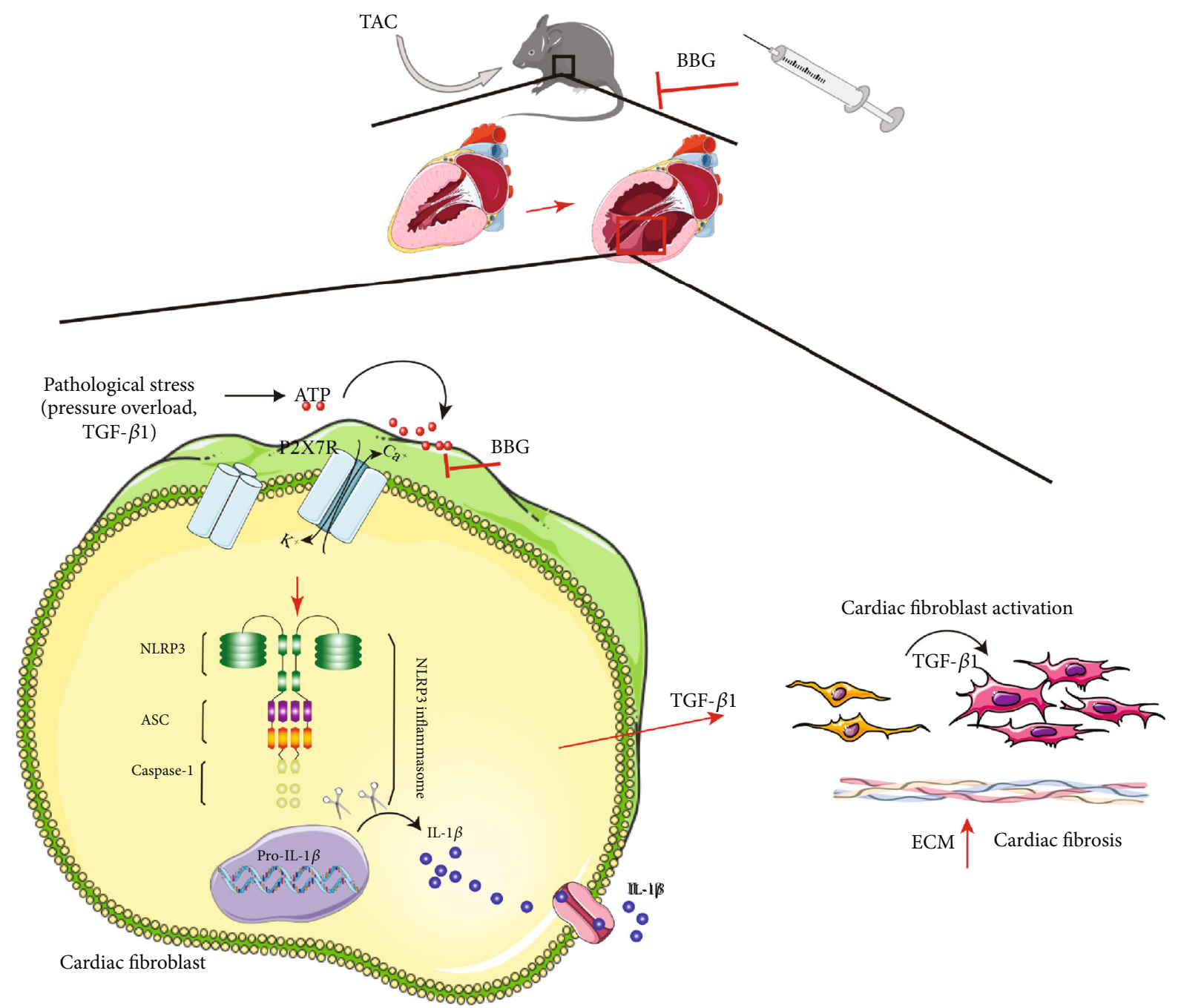

FIgURE 5: A schematic illustration of the mechanism of P2X7R in pressure overload-induced cardiac fibrosis and TGF- $\beta 1$-induced CF activation. In TAC-induced injury, pathological stress, such as pressure overload and TGF- $\beta 1$, triggers the release of ATP. Elevated ATP activates P2X7 receptors, contributing to P2X7-mediated NLRP3 inflammasome (NLRP3, ASC, and caspase-1) activation. This induces the release of IL- $1 \beta$, causing severe inflammation that precipitates cardiac fibrosis and aggravates TGF- $\beta 1$-induced $\mathrm{CF}$ activation. Moreover, inhibition of P2X7R with BBG inhibitor in TAC mice or CFs primed with TGF- $\beta 1$ reduces fibrotic extracellular matrix (ECM) gene expression and cardiac fibrosis.

antagonist (IL-1-ra) inhibited cardiac inflammation and improved systolic function. Elsewhere, it was reported that the genetic ablation of Nlrp3 in CNTg mice reduced IL-1 $\beta$ and caspase-1 expression thereby improving cardiac function [12]. Surprisingly, mice with fibroblast-specific deletion of IL-1 receptor-1 showed improved cardiac function and lower expression of remodeling markers after MI compared with littermate controls. These results illustrate that inflammasome activation and IL- $1 \beta$ production contribute to cardiac dysfunction and abnormal morphology, especially in CFs [41]. In agreement with previous works, we showed that NLRP3 inflammasome was activated in response to TAC [42]. Most importantly, we showed that inhibition of P2X7R with BBG significantly ameliorated cardiac fibrosis and downregulated NLRP3, IL- $1 \beta$, and caspase- 1 . A previous study indicated that the P2X7R antagonist ameliorated cardiac remodeling and prevented inflammasome formation following acute myocar- dial infarction (AMI) in mice [29]. Interestingly, other studies have shown that inflammasome formation occurs in CFs after cardiac injury which leads to the secretion of IL- $1 \beta$ [43]. Similarly, murine CFs exposed to lipopolysaccharide and ATP released IL- $1 \beta$, indicating the involvement of P2X7R in CF activation [28]. In our study, the knockdown of P2X7R ameliorated CF activation and suppressed NLRP3/IL-1 $\beta$ pathway, as evidenced by reduced periostin, $\alpha$-SMA, CTGF, NLRP3, and IL-1 $\beta$ levels. This is consistent with an earlier report in which P2X7R inhibitor or genetic deletion of P2X7R reduced the levels of IL-1 $\beta$ and NLRP3 in hepatic stellate cells treated with acetaldehyde, in cellular models of chronic alcoholic liver fibrosis [44]. Based on these observations, we speculate that the downregulation of P2X7R might inhibit the NLRP3/IL- $1 \beta$ pathway.

However, there are several limitations in this work. Although BBG is the most widely used P2X7R blocker, using 
P2X7R KO mice may more precisely demonstrate the function of P2X7R in cardiac fibrosis. Moreover, several lines of evidence have demonstrated that $\mathrm{P} 2 \mathrm{X} 7 \mathrm{R}$ expression is increased following activation of inflammatory cells such as macrophages, dendritic cells, mast cells, and T-lymphocytes $[43,45,46]$. Given that CFs are the principal producers of ECM, complex interactions between immune cells and CFs deserve further exploration.

We conclude that $\mathrm{P} 2 \mathrm{X} 7 \mathrm{R}$ activation promotes cardiac fibrosis. Also, we showed that inhibition of P2X7R may protect against $\mathrm{CF}$ activation and cardiac fibrosis by modulating the NLRP3/IL-1 $\beta$ pathway.

\section{Data Availability}

The accessibility data used to support the findings of this study were collected according with scientific research criteria and can be available from the corresponding author upon request.

\section{Conflicts of Interest}

The authors declare that they have no conflicts of interest..

\section{Authors' Contributions}

Junteng Zhou, Geer Tian, and Yue Quan collected the experimental data and wrote the manuscript. Xiaojiao Wang, Wenchao $\mathrm{Wu}$, and Miaoling Li contributed some materials. Xiaojing Liu supervised the whole project. Junteng Zhou and Geer Tian contributed equally to this work and should be considered co-first authors.

\section{Acknowledgments}

This study was funded by the Chinese National Natural Science Foundation (grant no. 11672197 accorded to Xiaojing Liu). We thank Dr. Xiaoping Gao and Dr. Jun Liu for providing assistance in echocardiography analysis.

\section{Supplementary Materials}

Figure S1: the mRNA level of pannexin1 in TAC- and shamoperated group. (Supplementary materials)

\section{References}

[1] G. A. Roth, C. Johnson, A. Abajobir et al., "Global, Regional, and National Burden of Cardiovascular Diseases for 10 Causes, 1990 to 2015," Journal of the American College of Cardiology, vol. 70, no. 1, pp. 1-25, 2017.

[2] M. Xie, J. S. Burchfield, and J. A. Hill, "Pathological ventricular remodeling: therapies: part 2 of 2," Circulation, vol. 128, no. 9, pp. 1021-1030, 2013.

[3] R. C. Lyon, F. Zanella, J. H. Omens, and F. Sheikh, "Mechanotransduction in cardiac hypertrophy and Failure," Circulation Research, vol. 116, no. 8, pp. 1462-1476, 2015.

[4] N. G. Frangogiannis, "Cardiac fibrosis: Cell biological mechanisms, molecular pathways and therapeutic opportunities," Molecular Aspects of Medicine, vol. 65, pp. 70-99, 2019.
[5] A. L. Birkenfeld, J. Jordan, M. Dworak, T. Merkel, and G. Burnstock, "Myocardial metabolism in heart failure: Purinergic signalling and other metabolic concepts," Pharmacology \& Therapeutics, vol. 194, pp. 132-144, 2019.

[6] T. Novitskaya, E. Chepurko, R. Covarrubias et al., "Extracellular nucleotide regulation and signaling in cardiac fibrosis," Journal of Molecular and Cellular Cardiology, vol. 93, pp. 4756, 2016.

[7] G. Vassort, "Adenosine 5'-Triphosphate: a P2-purinergic agonist in the Myocardium," Physiological Reviews, vol. 81, no. 2, pp. 767-806, 2001.

[8] A. Gombault, L. Baron, and I. Couillin, "ATP release and purinergic signaling in NLRP3 inflammasome activation," Frontiers in Immunology, vol. 3, 2013.

[9] C. Banfi, S. Ferrario, O. De Vincenti et al., "P2 receptors in human heart: upregulation of P2X6 in patients undergoing heart transplantation, interaction with $\mathrm{TNF} \alpha$ and potential role in myocardial cell death," Journal of Molecular and Cellular Cardiology, vol. 39, no. 6, pp. 929-939, 2005.

[10] E. Adinolfi, A. L. Giuliani, E. De Marchi, A. Pegoraro, E. Orioli, and F. Di Virgilio, "The P2X7 receptor: A main player in inflammation,” Biochemical Pharmacology, vol. 151, pp. 234244, 2018.

[11] G. Burnstock, "P2X ion channel receptors and inflammation," Purinergic Signal, vol. 12, no. 1, pp. 59-67, 2016.

[12] N. A. Bracey, P. L. Beck, D. A. Muruve et al., “The Nlrp3 inflammasome promotes myocardial dysfunction in structural cardiomyopathy through interleukin-1 $\beta$," Experimental Physiology., vol. 98, no. 2, pp. 462-472, 2013.

[13] K. Barth, C. Pfleger, A. Linge et al., "Increased P2X7R expression in atrial cardiomyocytes of caveolin-1 deficient mice," Histochemistry and Cell Biology, vol. 134, no. 1, pp. 31-38, 2010.

[14] G. Tu, G. Li, H. Peng et al., "P2X(7) inhibition in stellate ganglia prevents the increased sympathoexcitatory reflex via sensory-sympathetic coupling induced by myocardial ischemic injury," Brain Research Bulletin, vol. 96, pp. 7185, 2013.

[15] J. Liu, G. Li, H. Peng et al., "Sensory-sympathetic coupling in superior cervical ganglia after myocardial ischemic injury facilitates sympathoexcitatory action via P2X7 receptor," Purinergic Signal, vol. 9, no. 3, pp. 463-479, 2013.

[16] H. Zempo, Y. Sugita, M. Ogawa, R. Watanabe, J. I. Suzuki, and $\mathrm{M}$. Isobe, "A P2X7 receptor antagonist attenuates experimental autoimmune myocarditis via suppressed myocardial CD4 $+\mathrm{T}$ and macrophage infiltration and NADPH oxidase $2 / 4$ expression in mice," Heart and Vessels., vol. 30, no. 4, pp. 527-533, 2015.

[17] T. Furihata, S. Kinugawa, S. Takada et al., "The experimental model of transition from compensated cardiac hypertrophy to failure created by transverse aortic constriction in mice," Int J Cardiol Heart Vasc., vol. 11, pp. 24-28, 2016.

[18] J. Li, W. Wu, Y. Xin, M. Zhao, and X. Liu, "Inhibition of NogoB promotes cardiac hypertrophy via endoplasmic reticulum stress," Biomedicine \& Pharmacotherapy, vol. 104, pp. 193203, 2018

[19] Y. Xin, W. Wu, J. Qu et al., "Inhibition of Mitofusin-2 Promotes Cardiac Fibroblast Activation via the PERK/ATF4 Pathway and Reactive Oxygen Species," Oxidative Medicine and Cellular Longevity, vol. 2019, Article ID 3649808, 16 pages, 2019. 
[20] M. Zhao, L. Lu, S. Lei et al., "Inhibition of Receptor Interacting Protein Kinases Attenuates Cardiomyocyte Hypertrophy Induced by Palmitic Acid," Oxidative Medicine and Cellular Longevity, vol. 2016, Article ID 1451676, 13 pages, 2016.

[21] A. C. Morandini, L. E. Savio, and R. Coutinho-Silva, “The role of P2X7 receptor in infectious inflammatory diseases and the influence of ectonucleotidases," Biomed J., vol. 37, no. 4, pp. 169-177, 2014.

[22] M. J. L. Bours, E. L. R. Swennen, F. Di Virgilio, B. N. Cronstein, and P. C. Dagnelie, "Adenosine 5'-triphosphate and adenosine as endogenous signaling molecules in immunity and inflammation," Pharmacology \& Therapeutics, vol. 112, no. 2, pp. 358-404, 2006.

[23] G. Burnstock and G. E. Knight, "Cellular distribution and functions of P2 receptor subtypes in different systems," International Review of Cytology, vol. 240, pp. 31-304, 2004.

[24] L. C. Monção-Ribeiro, D. S. Faffe, P. T. Santana et al., "P2X7 receptor modulates inflammatory and functional pulmonary changes induced by silica," PLoS One, vol. 9, no. 10, article e110185, 2014.

[25] H.-C. Tung, F.-Y. Lee, S.-S. Wang et al., "The beneficial effects of P2X7 antagonism in rats with bile duct ligation-induced Cirrhosis," PLoS One, vol. 10, no. 5, article e0124654, 2015.

[26] J. Yin, Y. Wang, H. Hu et al., "P2X7receptor inhibition attenuated sympathetic nerve sprouting after myocardial infarctionviathe NLRP3/IL-1 $\beta$ pathway," Journal of Cellular and Molecular Medicine, vol. 21, no. 11, pp. 2695-2710, 2017.

[27] X. Ji, Y. Naito, H. Weng, K. Endo, X. Ma, and N. Iwai, “P2X7 deficiency attenuates hypertension and renal injury in deoxycorticosterone acetate-salt hypertension," American Journal of Physiology. Renal Physiology, vol. 303, no. 8, pp. F1207F1215, 2012.

[28] O. Sandanger, T. Ranheim, L. E. Vinge et al., "The NLRP3 inflammasome is up-regulated in cardiac fibroblasts and mediates myocardial ischaemia-reperfusion injury," Cardiovascular Research, vol. 99, no. 1, pp. 164-174, 2013.

[29] E. Mezzaroma, S. Toldo, D. Farkas et al., "The inflammasome promotes adverse cardiac remodeling following acute myocardial infarction in the mouse," Proceedings of the National Academy of Sciences of the United States of America, vol. 108, no. 49, pp. 19725-19730, 2011.

[30] H. Musa, J. O. Tellez, N. J. Chandler et al., "P2 purinergic receptor mRNA in rat and human sinoatrial node and other heart regions," Naunyn-Schmiedeberg's Archives of Pharmacology, vol. 379, no. 6, pp. 541-549, 2009.

[31] R. Fusco, E. Gugliandolo, F. Biundo, M. Campolo, R. di Paola, and S. Cuzzocrea, "Inhibition of inflammasome activation improves lung acute injury induced by carrageenan in a mouse model of pleurisy," The FASEB Journal, vol. 31, no. 8, pp. 3497-3511, 2017.

[32] H. A. Abdelaziz, M. E. Shaker, M. F. Hamed, and N. M. Gameil, "Repression of acetaminophen-induced hepatotoxicity by a combination of celastrol and brilliant blue G," Toxicology Letters, vol. 275, pp. 6-18, 2017.

[33] L. Duan, G. Hu, Y. Li, C. L. Zhang, and M. Jiang, "P2X7 receptor is involved in lung injuries induced by ischemiareperfusion in pulmonary arterial hypertension rats," Molecular Immunology., vol. 101, pp. 409-418, 2018.

[34] G. Tu, L. Zou, S. Liu et al., "Long noncoding NONRATT021972 siRNA normalized abnormal sympathetic activity mediated by the upregulation of P2X7 receptor in superior cervical ganglia after myocardial ischemia," Purinergic Signal, vol. 12, no. 3, pp. 521-535, 2016.

[35] F. di Virgilio, D. D. Ben, A. C. Sarti, A. L. Giuliani, and S. Falzoni, "The P2X7 Receptor in Infection and Inflammation," Immunity, vol. 47, no. 1, pp. 15-31, 2017.

[36] E. Dolmatova, G. Spagnol, D. Boassa et al., "Cardiomyocyte ATP release through pannexin 1 aids in early fibroblast activation," American Journal of Physiology. Heart and Circulatory Physiology, vol. 303, no. 10, pp. H1208-H1218, 2012.

[37] D. Lu and P. A. Insel, "Hydrolysis of extracellular ATP by ectonucleoside triphosphate diphosphohydrolase (ENTPD) establishes the set point for fibrotic activity of cardiac fibroblasts," The Journal of Biological Chemistry, vol. 288, no. 26, pp. 19040-19049, 2013.

[38] F.-L. Xiang, M. Fang, and K. E. Yutzey, "Loss of $\beta$-catenin in resident cardiac fibroblasts attenuates fibrosis induced by pressure overload in mice," Nature Communications, vol. 8, no. 1, p. 712, 2017.

[39] M. Nishida, Y. Sato, A. Uemura et al., "P2Y6 receptor-G $\alpha 12 / 13$ signalling in cardiomyocytes triggers pressure overloadinduced cardiac fibrosis," The EMBO Journal, vol. 27, no. 23, pp. 3104-3115, 2008.

[40] M. Gu, A. B. Zheng, J. Jin et al., "Cardioprotective Effects of Genistin in Rat Myocardial Ischemia-Reperfusion Injury Studies by Regulation of P2X7/NF- $\kappa \mathrm{B}$ Pathway," Evidence-based Complementary and Alternative Medicine, vol. 2016, 9 pages, 2016.

[41] S. A. Bageghni, K. E. Hemmings, N. Y. Yuldasheva et al., "Fibroblast-specific deletion of IL-1 receptor-1 reduces adverse cardiac remodeling following myocardial infarction," JCI Insight, vol. 4, no. 17, 2019.

[42] T. Suetomi, A. Willeford, C. S. Brand et al., "Inflammation and NLRP3 Inflammasome activation initiated in response to pressure overload by $\mathrm{Ca} 2+/$ Calmodulin-Dependent protein kinase II $\delta$ signaling in cardiomyocytes are essential for adverse cardiac Remodeling," Circulation, vol. 138, no. 22, pp. 2530 2544, 2018.

[43] M. Kawaguchi, M. Takahashi, T. Hata et al., "Inflammasome activation of cardiac fibroblasts is essential for myocardial ischemia/reperfusion injury," Circulation, vol. 123, no. 6, pp. 594-604, 2011.

[44] L. Shan, T. Jiang, L. Ci, Z. Liu, X. Lv, and J. Li, "Purine signaling regulating HSCs inflammatory cytokines secretion, activation, and proliferation plays a critical role in alcoholic liver disease," Molecular and Cellular Biochemistry, vol. 466, no. 1-2, pp. 91-102, 2020.

[45] Y. Wu, Y. Li, C. Zhang et al., "S100a8/a9 released by CD11b +Gr1+ neutrophils activates cardiac fibroblasts to initiate angiotensin II-induced cardiac inflammation and injury," Hypertension, vol. 63, no. 6, pp. 1241-1250, 2014.

[46] J. S. Wiley, R. Sluyter, B. J. Gu, L. Stokes, and S. J. Fuller, "The human P2X7 receptor and its role in innate immunity," Tissue Antigens, vol. 78, no. 5, pp. 321-332, 2011. 